\title{
Morfología dental y cénit gingival en adolescentes en la región Junin
}

Dental morphology and gingival zenith in teenagers from Junin region

\author{
Sixto García ${ }^{1, a}$, Adriana Torres ${ }^{1, b}$, Aylín Baltodano ${ }^{1, c}$
}

\section{RESUMEN}

La estética en la odontología ha cobrado mayor importancia, se han desarrollado diversos estudios antropométricos sobre estética, pero éstos se desarrollaron en poblaciones caucásicas. Objetivo: determinar la relación del cénit gingival y la morfología dentaria de las piezas dentales superiores del sector anterior en adolescentes peruanos. Material y Métodos: El estudio fue de tipo descriptivo, correlacional y transversal. La muestra fue de tipo probabilístico por conveniencia, y estuvo conformado por 92 adolescentes en la región Junín, Perú. Cada modelo fue analizado y se registró la morfología dental, la posición del cénit gingival y la distancia del cénit gingival al eje longitudinal de pieza dentaria. Resultados: Se obtuvo que la media de la ubicación del cénit gingival para el incisivo central superior era $72,8 \%$ hacia distal $(0,54 \mathrm{~mm})$, en cambio el incisivo lateral $(59,2 \%)$ y canino $(50,5 \%)$ estaban en la misma posición que el eje longitudinal del diente. Con respecto al tipo de forma dental, la forma oval se halló en el 71,2\% de los incisivos centrales, en el 67,9\% de los incisivos laterales y en el 73,9\% de los caninos. Se concluyó que la ubicación del cénit gingival en los incisivos centrales superiores se hallaba hacia distal en relación al eje longitudinal, con una distancia promedio de 0,54 mm, en cambio en los incisivos laterales y caninos superiores su cénit gingival se hallaba en el eje longitudinal del diente. Con respecto a la morfología dentaria la forma oval fue la más predominante. Conclusiones: Existe una migración hacia distal de los cénits gingivales en los incisivos centrales, pero no en los incisivos laterales o caninos superiores.

PALABRAS CLAVE: Cénit gingival, morfología dental, eje longitudinal, antropometría.

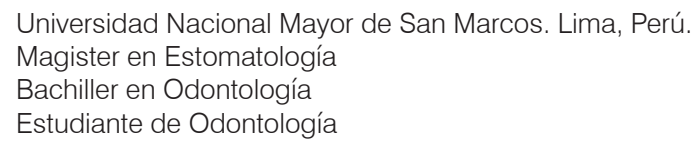




\begin{abstract}
Aesthetics in dentistry has gained greater importance, various anthropometric studies of aesthetics parameters have been developed, but these were developed in Caucasian populations. Objective: to determine the relationship of the gingival zenith and the dental morphology of the maxillary teeth of the anterior sector in Peruvian adolescents. Material and Methods: The study was descriptive, correlational and cross-sectional. The sample was of the probabilistic type for convenience, and consisted of 92 adolescents in the Junín region, Peru. Each model was analyzed and the dental morphology, the position of the gingival zenith and the distance from the gingival zenith to the longitudinal axis of the tooth were recorded. Results: The study obtained that the mean location of the gingival zenith for the upper central incisor was $72.8 \%$ distally $(0.54 \mathrm{~mm})$, for lateral incisor was $59.2 \%$ and for upper canine was $50.5 \%$, located in the same position as the longitudinal axis of the tooth. Regarding the type of dental shape, the oval shape was found in $71.2 \%$ of upper central incisors, in $67.9 \%$ of upper lateral incisors and in $73.9 \%$ of the canines. Location of gingival zenith in the upper central incisors was distal in relation to the longitudinal axis, with an average distance of $0.54 \mathrm{~mm}$, whereas in upper lateral incisors and upper canines, their gingival zenith was on tooth longitudinal axis. About dental morphology, oval shape was the most predominant. Conclusions: There is a distal migration of gingival zenith in the central incisors, but not in the lateral incisors or upper canines.
\end{abstract}

KEY WORDS: Gingival zenith, dental morphology and longitudinal axis, anthropometry

\section{INTRODUCCIÓN}

El rostro es un área importante de comunicación no verbal de nuestro cuerpo, el rostro comunica, revela y exhibe instantáneamente emociones (1).

En el ser humano la sonrisa es una expresión inherente, aparece desde que nacemos como un reflejo a la sensación de placer. Además, es una de las primeras maneras en la cual se produce un vinculo de comunicación madre- hijo durante su cuidado(2). La sonrisa es un gesto saludable y armónico que a su vez toma gran importancia dentro del estereotipo físico y de la percepción que otras personas tienen de nuestra apariencia y personalidad; una sonrisa agradable y atractiva refleja un carácter de alegría y amistad. Por ende, en la percepción de la belleza, una sonrisa simétrica toma un rol importante (3-5). Una sonrisa estética se obtiene con la integración perfecta de los tejidos duros (dientes) y blandos (labios y tejido gingival) de la cavidad oral, así como también del componente facial que incluye el tejido duro y blando de la cara (6-8).

Si bien la estética y la belleza son valores subjetivos, a través de los años se han realizado diversos estudios que nos brindan parámetros en cada uno de los componentes de la sonrisa, pero sabemos que los factores étnicos, culturales y las preferencias individuales son algunos de los aspectos que influyen durante la práctica clínica(9-12), por ende, estos parámetros establecidos no se pueden aplicar completamente a nuestra realidad.

El objetivo del estudio fue relacionar la morfología dental y cénit gingival, dos componentes de una sonrisa estética, en nuestra sociedad de carácter multiétnico, y poder así establecer así, parámetros con base en nuestra sociedad. La siguiente investigación aportará a la comunidad odontológica los datos estadísticos de dos de los parámetros evaluados en estética dental: morfología dental y cenit gingival, y si existe relación entre ambas. Obteniendo datos más fidedignos a nuestra población y útil para la elaboración diagnóstica y tratamiento adecuado del paciente peruano.

\section{MATERIAL Y MÉTODOS}

El estudio fue de tipo descriptivo, correlacional y transversal. La muestra obtenida fue de tipo probabilístico por conveniencia, y estuvo conformado por 92 alumnos adolescentes del centro educativo nacional Santiago Antúnez de Mayolo del distrito de Pichanaki, Chanchamayo- Junín, cuya edad oscilaba entre $\operatorname{los} 12$ a 17 años de edad. 
Se les informó del estudio y se hicieron partícipes, con la autorización de sus padres a través de la firma del consentimiento informado, aquellos estudiantes que cumplieron con los criterios de inclusión y exclusión del estudio: presentar todas las piezas dentarias antero superiores ubicados correctamente, no haber recibido o estar en tratamiento ortodóntico, no presentar restauraciones a nivel cérvico-vestibular, no presentar gingivitis moderada o severa, ni presentar recesión gingival. Dentro del consentimiento informado se le adicionó una ficha de asentimiento informado para los alumnos participantes del estudio. A cada participante se le asignó una ficha de recolección de datos para el llenado de sus datos personales y se le tomó modelo de estudio codificado con la misma enumeración de la ficha para su identificación.

Cada modelo de estudio fue analizado, y mediante observación directa se definió el tipo de forma dental de cada pieza dental a estudiar. Con respecto al cénit gingival, con el uso de un calibrador digital INSIZE ${ }^{\circledR}$ se midió la longitud mesio-distal de cada pieza dental, mediante la división de dicho valor en dos, se obtuvo la posición por la cual pasaría el eje axial del diente. Además, se ubicó el punto más apical del contorno de la encía (cénit gingival) mediante observación directa al modelo de estudio, marcándole un punto para su evaluación. Un solo examinador realizó las observaciones despues de calibrarse con un experto en la ciudad de Lima.

Por último, se registró en la ficha de recolección de datos, la longitud entre el punto más apical del margen gingival y el eje axial de cada pieza dentaria, asimismo, la ubicación con respecto al lado en el que se encontraba dicho punto.
El procesamiento y análisis estadístico de los datos se realizó mediante el programa estadístico SPSS versión 15 . Se relacionó la ubicación del cénit gingival en cada pieza dental con la prueba estadística chi cuadrado, de igual manera para relacionar la posición del cénit gingival y la morfología dental con el tipo de pieza dentaria se aplicó la prueba de chi cuadrado. Se relacionó la distancia del cénit gingival entre mesial y distal según pieza dentaria con la prueba U de Mann-Whitney. Por último, se utilizó la prueba del chi cuadrado para relacionar la morfología dental en cada tipo de pieza dentaria.

\section{RESULTADOS}

Al análisis de los modelos de estudio se encontró que el cénit gingival del incisivo central se ubicaba en un $72,8 \%$ de la muestra hacia distal del eje longitudinal de la pieza observada, mientras que, en los incisivos laterales se ubícaba en el 59,2\% de la muestra, el cénit gingival se encontraba sobre el eje longitudinal del diente, al igual que en el $50,5 \%$ de los caninos (tabla 1 y figura 1 ), pero no se encontró relación entre la ubicación del cénit gingival y el género (tabla 2).

Al cuantificar la distancia en el que se ubica el cenit con respecto al eje longitudinal, se encontró que fue de $0,54 \mathrm{~mm}$ hacia distal en incisivos centrales (tabla 3), y $0 \mathrm{~mm}$ en los incisivos laterales y caninos superiores (figura 2). No se encontró relación entre la distancia del cénit al eje longitudinal con el género (tabla 4) y, asimismo, con el grupo etario (tabla 5).

En cuanto a la morfología dental, la mayoría de las piezas dentales analizadas tenían una forma oval,

Tabla 1. Ubicación del Cenit gingival en cada tipo de pieza dentaria del sector antero superior de los adolescentes de 12 a 17 años de edad de la IEN Santiago Antúnez de Mayolo, del distrito de Pichanaki, Chanchamayo, Junín, en el año 2013.

\begin{tabular}{llclllc}
\hline \multirow{2}{*}{ PIEZA DENTAL } & \multicolumn{5}{c}{ CÉNIT GINGIVAL } \\
\cline { 2 - 7 } & \multicolumn{3}{c}{ MESIAL } & & DISTAL & \multicolumn{2}{c}{ SOBRE EL EJE } \\
\hline I. central & $\mathrm{n}$ & $\%$ & $\mathrm{n}$ & $\%$ & $\mathrm{n}$ & $\%$ \\
& 4 & 2,2 & 134 & 72,8 & 46 & 25 \\
I. lateral & 7 & 3,8 & 68 & 37 & 109 & 59,2 \\
Canino & 65 & 35,3 & 26 & 14,1 & 93 & 50,5 \\
Prueba de chicuadrado & & & & &
\end{tabular}




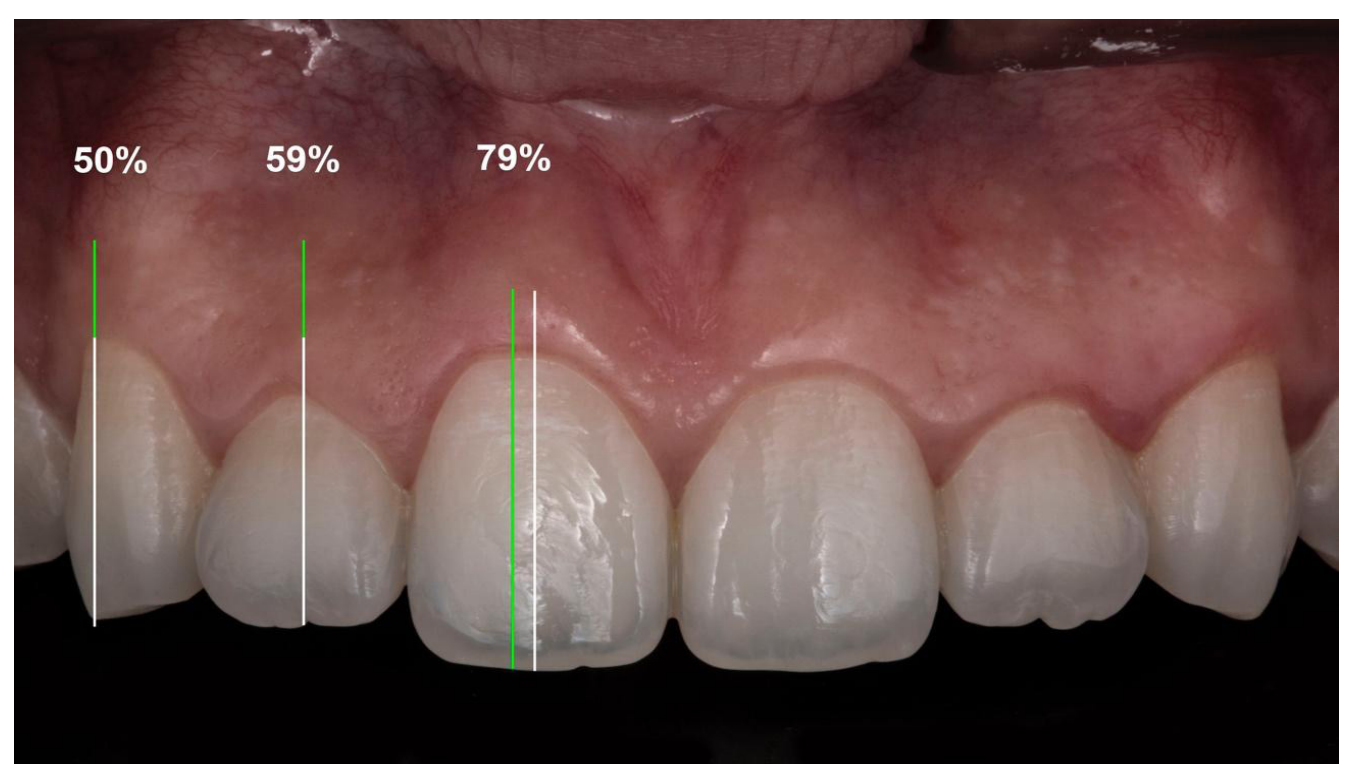

Figura 1. Porcentaje de ubicación del cenit gingival. El incisivo central $72 \%$ en los incisivos laterales el 59,2\% el eje longitudinal del diente y los caninos 50,5\%

Tabla 2. Cenit gingival en cada tipo de pieza dentaria del sector antero superior según grupo etario de los adolescentes de 12 a 17 años de edad de la IEN Santiago Antúnez de Mayolo, del distrito de Pichanaki, Chanchamayo, Junín, en el año 2013.

\begin{tabular}{lllllllll}
\hline \multirow{2}{*}{ PIEZA DENTAL } & CÉNIT GINGIVAL & \multicolumn{4}{c}{ GRUPO ETARIO } & \multicolumn{2}{c}{ TOTAL } \\
\cline { 3 - 6 } & & \multicolumn{3}{c}{$\mathbf{1 2 - 1 4}$ años } & $\mathbf{1 5 - 1 7}$ años & & \\
\cline { 3 - 7 } Incisivo Central & & $\mathrm{n}$ & $\%$ & $\mathrm{n}$ & $\%$ & $\mathrm{n}$ & $\%$ \\
\hline & Mesial & 1 & 1,7 & 3 & 2,4 & 4 & 2,2 \\
& Distal & 45 & 77,6 & 89 & 70,6 & 134 & 72,8 \\
& Sobre el eje & 12 & 20,7 & 34 & 27 & 46 & 25 \\
& Mesial & 4 & 6,9 & 3 & 2,4 & 7 & 3,8 \\
& Distal & 28 & 48,3 & 40 & 31,7 & 68 & 37 \\
& Sobre el eje & 26 & 44,8 & 83 & 65,9 & 109 & 59,2 \\
& Mesial & 21 & 36,2 & 44 & 34,9 & 65 & 35,3 \\
& Distal & 11 & 36,2 & 44 & 34,9 & 65 & 35,3 \\
& Sobre el eje & 26 & 44,8 & 67 & 53,2 & 93 & 50,5 \\
\hline
\end{tabular}

Prueba de chi cuadrado

Tabla 3. Distancia del cenit gingival al eje longitudinal de la pieza dentaria en el incisivo central de acuerdo a la ubicación del cenit gingival de los adolescentes de 12 a 17 años de edad de la IEN Santiago Antúnez de Mayolo, del distrito de Pichanaki, Chanchamayo, Junín, en el año 2013.

\begin{tabular}{lllllll}
\hline PIEZA DENTAL & \multicolumn{4}{c}{ CÉNIT GINGIVAL } \\
\hline \multirow{3}{*}{ I. central } & \multicolumn{4}{c}{ MESIAL } & \multicolumn{3}{c}{ DISTAL } \\
\cline { 2 - 7 } & $\mathrm{n}$ & Promedio & DS & $\mathrm{n}$ & Promedio & DS \\
\cline { 2 - 7 } & 4 & 0,40 & 0,12 & 134 & 0,54 & 0,28 \\
\hline Prueba U de Mann-Whitn & & & & &
\end{tabular}




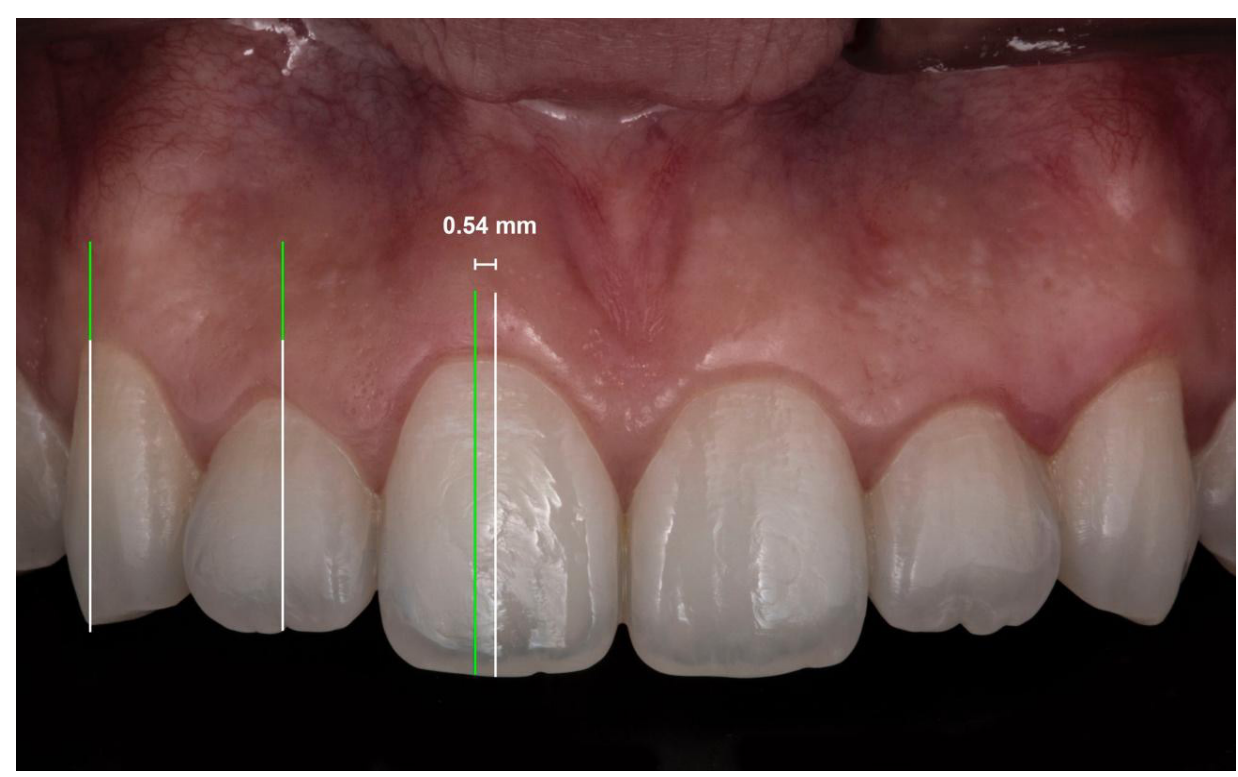

Figura 2: Distancia del cénit gingival al eje longitudinal de piezas dentarias del sector antero superior. El Incisivo central superior tenía un cenit con un promedio $0.54 \mathrm{~mm}$ de desviación hacia distal del eje axial.

Tabla 4. Distancia del cenit gingival al eje longitudinal en cada tipo de pieza dentaria según género de los adolescentes de 12 a 17 años de edad de la IEN Santiago Antúnez de Mayolo, del distrito de Pichanaki, Chanchamayo, Junín, en el año 2013.

\begin{tabular}{|c|c|c|c|c|c|c|c|}
\hline \multirow{3}{*}{$\begin{array}{l}\text { Tipo de } \\
\text { pieza } \\
\text { dentaria }\end{array}$} & \multirow{3}{*}{$\begin{array}{l}\text { Cénit } \\
\text { gingival }\end{array}$} & \multicolumn{6}{|c|}{ GENERO } \\
\hline & & \multicolumn{3}{|c|}{ FEMENINO } & \multicolumn{3}{|c|}{ MASCULINO } \\
\hline & & $\mathbf{n}$ & Promedio & DS & $\mathbf{n}$ & Promedio & DS \\
\hline \multirow{2}{*}{ IC } & M & 3 & 0,43 & 0,12 & 1 & $\mathrm{NC}$ & $\mathrm{NC}$ \\
\hline & $\mathrm{D}$ & 69 & 0,58 & 0,32 & 65 & 0,51 & 0,23 \\
\hline \multirow{2}{*}{ IL } & M & 4 & 0,38 & 0,35 & 3 & 0,73 & 0,75 \\
\hline & $\mathrm{D}$ & 33 & 0,41 & 0,20 & 35 & 0,37 & 0,20 \\
\hline \multirow{2}{*}{$\mathrm{C}$} & M & 36 & 0,55 & 0,32 & 29 & 0,46 & 0,25 \\
\hline & $\mathrm{D}$ & 8 & 0,33 & 0,28 & 18 & 0,29 & 0,11 \\
\hline
\end{tabular}

Prueba U de Mann-Whitney

$\mathrm{NC}=$ No calculable

Tabla 5. Distancia del cenit gingival al eje longitudinal en cada tipo de pieza dentaria según grupo etario de los adolescentes de 12 a 17 años de edad de la IEN Santiago Antúnez de Mayolo, del distrito de Pichanaki, Chanchamayo, Junín, en el año 2013.

\begin{tabular}{|c|c|c|c|c|c|c|c|}
\hline \multirow{3}{*}{$\begin{array}{l}\text { Tipo de pieza } \\
\text { dentaria }\end{array}$} & \multirow{3}{*}{ Cénit gingival } & \multicolumn{6}{|c|}{ GRUPO ETARIO } \\
\hline & & \multicolumn{3}{|c|}{ 12-14 años } & \multicolumn{3}{|c|}{ 15-17 años } \\
\hline & & $\mathbf{n}$ & Promedio & DS & $\mathbf{n}$ & Promedio & DS \\
\hline \multirow{2}{*}{ IC } & M & 1 & $\mathrm{NC}$ & $\mathrm{NC}$ & 3 & 0,43 & 0,12 \\
\hline & $\mathrm{D}$ & 45 & 0,56 & 0,35 & 89 & 0,53 & 0,24 \\
\hline \multirow{2}{*}{ IL } & M & 4 & 0,40 & 0,34 & 3 & 0,70 & 0,78 \\
\hline & $\mathrm{D}$ & 28 & 0,42 & 0,24 & 40 & 0,36 & 0,17 \\
\hline \multirow{2}{*}{$\mathrm{C}$} & M & 21 & 0,51 & 0,30 & 44 & 0,51 & 0,29 \\
\hline & $\mathrm{D}$ & 11 & 0,32 & 0,24 & 15 & 0,29 & 0,11 \\
\hline
\end{tabular}

Prueba U de Mann-Whitney

$\mathrm{NC}=$ No calculable 
seguido de cuadrado y, por último, triangular, todo ello ocurrió en las diversas piezas dentarias del sector antero superior: incisivo central, incisivo lateral y canino superiores (tabla 6).

Al relacionar la morfología dental y el cénit gingival, se obtuvo que, en los incisivos centrales superiores el cénit gingival se ubicó hacia distal en todos los tipos de morfología dental hallados; mientras que en los incisivos laterales superiores, éste se hallaba sobre el eje longitudinal en las piezas cuadradas y ovales, en cambio se ubicaba hacia distal en piezas triangulares, de igual manera ocurrió en los caninos superiores. (tabla 7).

\section{DISCUSIÓN}

Según los resultados del estudio, se halló que la ubicación del cenit gingival en el incisivo central superior estaba hacia distal en un $72,8 \%$, dicho hallazgo coincide con los estudios realizados por Nappe y col., (13) en Chile, Vera en Ecuador (14), Mattos y Santana (15) en Brasil y por Flores y col., (16) en Perú.

Caso contrario se registró en los incisivos laterales superiores, en los cuales, la ubicación del cénit gingival se hallaba sobre el eje longitudinal en un $59,2 \%$ de la muestra. Este hallazgo es similar a lo propuesto por Rufenacht (17), Moncada (18), Ahmad (19), y Cohen (20), quienes mencionan la coincidencia de ambos parámetros dentales. Por otro lado, Vera refuta esto, debido a que en su estudio obtuvo que la ubicación del cénit gingival en los incisivos laterales se encontraban hacia distal con respecto al eje longitudinal en el 100\% de su muestra (14).

La ubicación del cénit gingival en los caninos superiores, en un $50.5 \%$ de la muestra, se encontró

Tabla 6. Morfología dental de cada tipo de pieza dentaria de los adolescentes de 12 a 17 años de edad de la IEN Santiago Antúnez de Mayolo, del distrito de Pichanaki, Chanchamayo, Junín, en el año 2013.

\begin{tabular}{lllllll}
\hline PIEZA DENTAL & \multicolumn{5}{c}{ MORFOLOGÍA DENTAL } \\
\cline { 2 - 7 } & CUADRADO & \multicolumn{2}{c}{ OVAL } & \multicolumn{3}{c}{ TRIANGULAR } \\
\cline { 2 - 7 } & $\mathbf{n}$ & $\mathbf{\%}$ & $\mathbf{n}$ & $\mathbf{\%}$ & $\mathbf{n}$ & $\mathbf{\%}$ \\
\hline I. central & 45 & 24,5 & 131 & 71,2 & 8 & 4,3 \\
I. lateral & 53 & 28,8 & 125 & 67,9 & 6 & 3,3 \\
Canino & 44 & 23,9 & 136 & 73,9 & 4 & 2,2 \\
\hline
\end{tabular}

Prueba de chi cuadrado

Tabla 7. Morfología dental y cenit gingival en cada tipo de pieza dentaria de los adolescentes de 12 a 17 años de edad de la IEN Santiago Antúnez de Mayolo, del distrito de Pichanaki, Chanchamayo, Junín, en el año 2013.

\begin{tabular}{llllllll}
\hline PIEZA DENTAL & CÉNIT GINGIVAL & \multicolumn{3}{c}{ MORFOLOGÍA DENTAL } \\
\hline & & \multicolumn{3}{c}{ CUADRADO } & \multicolumn{1}{c}{ OVAL } & \multicolumn{2}{c}{ TRIANGULAR } \\
\cline { 3 - 8 } & & $\mathbf{n}$ & $\mathbf{\%}$ & $\mathbf{n}$ & $\mathbf{\%}$ & $\mathbf{n}$ & $\mathbf{\%}$ \\
\hline \multirow{3}{*}{ Incisivo Central } & Mesial & 0 & 0 & 4 & 3,1 & 0 & 0 \\
& Distal & 25 & 55,6 & 103 & 78,6 & 6 & 75,0 \\
& Sobre el eje & 20 & 44,4 & 24 & 18,3 & 2 & 25,0 \\
& Mesial & 2 & 3,8 & 5 & 4,0 & 0 & 0 \\
Incisivo Lateral & Distal & 19 & 35,8 & 45 & 36,0 & 4 & 66,7 \\
& Sobre el eje & 32 & 60,4 & 75 & 60,0 & 2 & 33,3 \\
& Mesial & 17 & 38,6 & 49 & 36,0 & 0 & 0 \\
Canino & Distal & 3 & 6,8 & 19 & 14,0 & 4 & 100,0 \\
& Sobre el eje & 24 & 54,5 & 68 & 50,0 & 0 & 0 \\
\hline
\end{tabular}

Prueba exacta de Fisher 
sobre el eje longitudinal dentario. Mattos y Santana (15), Vera (14), Chu y col., (21) coincidieron con dicho hallazgo pero obtuvieron porcentajes mayores, al igual que Flores y col., quienes obtuvieron en un $77,5 \%$ de su muestra en una población peruana pero con la diferencia que el estudio se desarrolló en un mayor grupo etario (16). Nappe y col., (13), en una población chilena, hallaron que el cénit gingival de los caninos superiores estaba a un promedio de 0,081 $\mathrm{mm}$ del eje longitudinal.

Con respecto a la ubicación de cénit gingival en los incisivos centrales superiores, en el $72,8 \%$ de la muestra se halló que se ubicaba hacia distal. Al cuantificar la distancia con respecto al eje longitudinal se obtuvo una media de $0,54 \mathrm{~mm}$. Estudios realizados por Chu y col., (21), Nappe y col., (13), y Vera(14) hallaron resultados similares, pero con una marcada diferencia con respecto a la distancia obtenida por Chu y col., que fue de $1 \mathrm{~mm}$ a distal (12), Nappe y col., fue de 1,419 mm (13), y de Vera fue de 0,89 $\mathrm{mm}$ (14). Esta diferencia en las longitudes puede ser consecuencia a la realización del estudio en diferentes poblaciones y diferentes grupos etarios.

En relación a la morfología dental, la forma predominante fue la forma oval en todas las piezas dentarias (50\%), este resultado fue similar al hallado por Moncada(18), con una mayor preponderancvia de la forma oval, al igual que Melo y col.,, los cuales mencionaron que en el $62,9 \%$ de su muestra presentó forma dentaria oval (22). Acosta y col., cuya muestra fue una población colombiana donde hallaron correlación entre la forma de los incisivos centrales superiores, la forma de los arcos dentarios y de la forma ovalada del contorno facial (23). La forma dental ovalada se encontró en el $50 \%$ de su muestra. Alvino muestra que el perfil facial leptoprosópicos presenta el mayor porcentaje y en ellos predomina la forma oval del incisivo central superior (24); dicho resultado fue diferente al obtenido del estudio realizado por Bellido en una población de adolescentes en la cuidad de Juliaca- Puno, en el cual el tipo de morfología dental de los incisivos centrales superiores era cuadrada (25). Estas diferencias, según Lombardi es debido principalmente al evidente mestizaje actual en la población mundial y las variadas posibilidades de combinar las formas de la cara con formas halladas en los incisivos centrales superiores (26). Podemos concluir que existe una migración hacia distal de los cénits gingivales en los incisivos centrales, pero no en los incisivos laterales o caninos superiores.

\section{Correspondencia}

Sixto García Linares

Avenida Brasil 2481 Dpto. 203 Jesus Maria, Lima, Perú.

Teléfono: 51993458760

Correo electrónico: sgarcial@unmsm.edu.pe

\section{REFERENCIAS BIBLIOGRAFÍCAS}

1. Palma C. Análisis de la percepción estética de la sonrisa. Tesis de pregrado. Santiago de Chile: Universidad de Chile; 2010.

2. Hernández M. La sonrisa y su patología. Rev Mex Med Física y Rehabil. 2000; 3:49-52.

3. Martinez $\mathrm{O}$, Peñarrocha $M$. Sonrisas del mundo. Valencia: Universitat de Valencia; 2012.

4. Londoño MA, Botero P. La sonrisa y sus dimensiones. Rev Fac Odontol Univ Antioquia. 2012; 23(2):353-65.

5. Balsells E, Camps D, Ustre J. Evaluación de la sonrisa. An Odontoestomatol. 1996; 1:36-9.

6. Bhuvaneswaran M. Principles of smile design. J Conserv Dent. 2010;13(4):225-32.

7. García S. Nueva Clasificación de la enfermedad periodontal. Odontol Sanmarquina. 2003; 6(11): 4850 .

8. Garcia S, Orrego G. Parámetros estéticos del perfil facial: El Ángulo Nasolabial. Kiru. 2014; 11(1):86-9.

9. Sandeep N., Satwalekar P., Srinivas S, Sekhar C, Ramaswamy G, Anantha B. An analysis of maxillary anterior teeth dimensions for the existence of golden proportion: Clinical study. J Int Oral Heal. 2015;7(9):1821.

10. Curtis D, Lin G-H, Fishman A, et al. Patient-centered risk assessment in implant treatment planning. The International Journal of Oral \& Maxillofacial Implants. 2019.34: 506-520.

11. Garcia S, Maldonado L, Reyes M, Chamorro K, Alata $\mathrm{S}$, Gejaño A. IADR Abstract archives gingival tissue width and color changes of gingival margin caused by restorative materials: An In Vitro Study. J Dent Res. 2017; 96:3540-2017.

12. Howe MS, Keys W, Richards D. Long-term (10-year) dental implant survival: A systematic review and sensitivity meta-analysis. J Dent. 2019; 84:9-21.

13. Nappe C, Donoso F, Díaz I, Díaz J. Parámetros mucogingivales y dentarios en estudiantes de odontología chilenos. Rev Clin Periodoncia Implant Rehabil Oral. 2015; 8(3): 228-33.

14. Vera M, Molina D. Zénit, línea y nivel gingival en los estudiantes de la Facultad de Odontología de la 
Universidad de Cuenca. Cuenca: Universidad de Cuenca; 2018.

15. Mattos C, Santana R. A quantitative evaluation of the spatial displacement of the gingival zenith in the maxillary anterior dentition. J Periodontol. 2008; 79(10):1880-5.

16. Flores-Jara P, Delgado-Cotrina L, Webb-Linares J, Evangelista-Alva A. Estudio comparativo de dos instrumentos para determinar la ubicación del cenit gingival en dientes anterosuperiores. Rev Estomatol Hered. 2013;23(3):133-41.

17. Rufenacht C. Fundamentals of Esthetics. Berne: Quintessence Publishing; 1990. p. 1-372.

18. Moncada G. Parámetros para la evaluación de la estética dentaria antero superior. Rev Dent Chile. 2008; 99(3):29-38.

19. Ahmad I. Anterior dental aesthetics: Gingival perspective. Br Dent J. 2005; 199(4):195-202.

20. Cohen E. Atlas of Cosmetic and Reconstructive Periodontal Surgery. Shelton, Connecticut: People's Medical Publishing House; 2009.

21. Chu S, Tan J, Stappert C, Tarnow D. Gingival Zenith Positions and Levels of the Maxillary Anterior Dentition. J Esthet Restor Dent. 2009;21(2):113-20.
22. Melo M, Ata-Ali J, Ata-Ali F, et al. Evaluation of the maxillary midline, curve of the upper lip, smile line and tooth shape: A prospective study of 140 Caucasian patients. BMC Oral Health. 2020; 20(1). Doi: 10.1186/ s12903-020-1031-y

23. Acosta D, Porras A, Moreno F. Relación entre la forma del contorno facial, los arcos dentarios e incisivos centrales superiores en estudiantes de odontología de la Universidad del Valle en Cali. Rev estomat salud. 2011; 19(1):8-13.

24. Alvino M. Análisis de la sonrisa en relación a las proporciones faciales en pacientes jóvenes de 17 a 20 años. Lima: Universidad Nacional Federico Villarreal; 2009.

25. Bellido P. Relación entre biotipo facial, forma de arcos dentarios y forma de incisivos centrales superiores en estudiantes de 16 años de la Institución Educativa Emblemática G. U. E. José Antonio Encinas- Juliaca. Puno: Universidad Nacional del Altiplano; 2016.

26. Lombardi RE. The principles of visual perception and their clinical application to denture esthetics. J Prosthet Dent. 1973;29(4):358-82.

Recibido : 05-02-2021

Aceptado : 26-06-2021 